Article (refereed)

Kay, A. L.; Davies, H. N.; Bell, V. A.; Jones, R. G. 2009 Comparison of uncertainty sources for climate change impacts: flood frequency in England. Climatic Change, 92 (1-2). 41-63. doi:10.1007/s10584-008-9471-4

(C) Springer

This version available at http://nora.nerc.ac.uk/3097/

NERC has developed NORA to enable users to access research outputs wholly or partially funded by NERC. Copyright and other rights for material on this site are retained by the authors and/or other rights owners. Users should read the terms and conditions of use of this material at http://nora.nerc.ac.uk/policies.html\#access

This document is the author's final manuscript version of the journal article, incorporating any revisions agreed during the peer review process. Some differences between this and the publisher's version remain. You are advised to consult the publisher's version if you wish to cite from this article.

http://www.springer.com/ 


\title{
Comparison of uncertainty sources for climate change impacts: flood frequency in England
}

\author{
Kay, A.L. ${ }^{1}$, Davies, H.N. ${ }^{1}$, Bell, V.A. ${ }^{1}$ and Jones, R.G. ${ }^{2}$ \\ ${ }^{1}$ Centre for Ecology and Hydrology, Maclean Building, Crowmarsh Gifford, \\ Wallingford, Oxfordshire, OX10 8BB, UK \\ ${ }^{2}$ Met Office Hadley Centre (Reading Unit), Meteorology Building, University of \\ Reading, Reading, RG6 6BB, UK
}

Correspondence to: A.L. Kay (alkay@ceh.ac.uk)

\begin{abstract}
This paper investigates the uncertainty in the impact of climate change on flood frequency in England, through the use of continuous simulation of river flows. Six different sources of uncertainty are discussed: future greenhouse gas emissions; Global Climate Model (GCM) structure; downscaling from GCMs (including Regional Climate Model structure); hydrological model structure; hydrological model parameters and the internal variability of the climate system (sampled by applying different GCM initial conditions). These sources of uncertainty are demonstrated (separately) for two example catchments in England, by propagation through to flood frequency impact. The results suggest that uncertainty from GCM structure is by far the largest source of uncertainty. However, this is due to the extremely large increases in winter rainfall predicted by one of the five GCMs used. Other sources of uncertainty become more significant if the results from this GCM are omitted, although uncertainty from sources relating to modelling of the future climate is generally still larger than that relating to emissions or hydrological modelling. It is also shown that understanding current and future natural variability is critical in assessing the importance of climate change impacts on hydrology.
\end{abstract}

\section{Keywords}

Climate change; uncertainty; hydrological impacts; flood frequency.

\section{Introduction}

There is uncertainty in the results of any modelling, of different types and from different sources. Any individual source of uncertainty, if quantified in some way, can be propagated through to give an uncertainty in the end result. This propagation could be done individually, for each different source of uncertainty, (termed here 'singlepropagation') or in combination with other sources (termed here 'multi-propagation'). It is possible that some uncertainties can be reduced, through research or modelling improvements, and the quantification and propagation of uncertainty sources could allow research to be targeted at specific areas where uncertainty is currently large but potentially reducible. However, some uncertainties cannot currently be reduced and it is unlikely that uncertainty can ever be completely removed. In the case where modelling results are presented with some quantification of (currently irreducible) uncertainty, the end-user of the results must decide how best to take account of that uncertainty within 
the decision-making process. Proper quantification of uncertainty is vital to facilitate a risk-based approach to decision-making.

This paper considers various sources of uncertainty which apply in hydrological modelling for flood frequency estimation under climate change, using data available from various Global and Regional Climate Models (GCMs and RCMs). There are many sources of uncertainty in a climate change impact study, and this paper does not claim to cover all of them comprehensibly. However, it does demonstrate and compare the uncertainty in the flood frequency impact for a large number of possible sources (for two example catchments in England); more than any previous studies of which we are aware. The sources of uncertainty are described in more detail below. Those being considered are: future greenhouse gas emissions; GCM structure; downscaling from GCMs (including RCM structure); hydrological model structure; hydrological model parameters. The effect of the internal variability of the climate system is also considered. The study uses the PDM rainfall-runoff model (Moore 1985, 2007), and all modelling is done for the present and for the 2080s time-slice (2071-2100).

Several studies have looked at the effect of different sources of uncertainty on precipitation, and greater uncertainties in precipitation might be expected to lead to greater uncertainties in flows. Rowell (2006) used PRUDENCE data (http://prudence.dmi.dk) to compare the effect of different sources of uncertainty (emissions scenario, GCM, RCM, and initial condition ensemble) on changes in seasonal surface air temperature (SAT) and precipitation over the UK. For both variables, and in all seasons, it was found that uncertainty from GCM formulation was the largest. Uncertainty from the RCM formulation was found to be largest in the summer, but similar in size to that from internal variability and, for precipitation, greater that than due to choice of emissions scenario. Other work using PRUDENCE data includes propagation through hydrological models to river flows (Graham et al. 2007a, b) and also demonstrates larger uncertainties due to choice of GCM as against RCM or emissions scenario. However, it should be noted that the uncertainty from GCM formulation is unlikely to represent the full range of possibilities in the PRUDENCE work, as data were only available from two different GCMs (with the same nested RCM). Similarly, emissions uncertainty will be underestimated as data were only available from two scenarios (A2 and B2), which do not span the full SRES range (IPCC, 2000). Studies of changes in global mean rainfall from different GCMs and emissions scenarios suggest that GCM uncertainty dominates emissions uncertainty (Jenkins and Lowe, 2003).

Previous work on uncertainty in the impact of climate change on flooding includes that of Reynard et al. (2004), for 10 British catchments, which finds that emissions uncertainty (four UKCIP02 scenarios; see Section 3.1) is less important than downscaling uncertainty. Cameron (2006) also uses the UKCIP02 scenarios, for a catchment in Scotland, as well as two additional scenarios ('H-wet' and 'H-dry') based on the range of GCM uncertainty for the High emissions scenario. He also considers natural variability, through the use of a stochastic rainfall model, and finds that the range of changes from the additional ' $\mathrm{H}$-wet' scenario is beyond the range of current natural variability, whereas changes under the other five scenarios show a significant overlap with current natural variability. This emphasises both the need to consider more 
than one GCM (with GCM uncertainty suggested to be much wider than emissions uncertainty), and to put the changes in context with a demonstration of natural variability. The latter was also demonstrated by Cameron et al. (2000) for the flood frequency of a catchment in Wales, and by Arnell (2003) for mean monthly flows of six catchments in Britain. Booij (2005) found, for changes in flood frequency for a large river basin in Western Europe, that the uncertainty due to natural variability is potentially larger than that due to the use of data from different climate models (3 GCMs and 2 RCMs), but that the uncertainty due to hydrological model structure (different spatial resolutions of one model) or parameterisation is less important.

Recent work looking at the uncertainty in the impact of climate change on water resources (monthly mean flows) for four catchments in Britain (Prudhomme and Davies 2008) suggested that GCM uncertainty (from 3 GCMs) was the largest source of uncertainty, with downscaling uncertainty also significant. Hydrological uncertainty was found to vary significantly between catchments. Emissions uncertainty was not found to be significant, but only two emissions scenarios (A2 and B2) were used and the time-slice under consideration was the 2020s; emissions uncertainty will be higher for later time-slices. A European-scale study of changes in mean runoff for the 2050s (Arnell, 1999) also concluded that, in general, the greatest uncertainty comes from GCMs, with less from hydrological modelling (although the impact in some parts of mainland Europe showed high sensitivity to the value of a particular model parameter). Wilby (2005) investigated the impact of climate change on monthly mean flows for the Thames, particularly looking at the effect of parameter uncertainty from the conceptual water balance model. He found that calibration uncertainty was comparable in size to emissions uncertainty (even for the 2080s), although only two emissions scenarios (A2 and B2) were used. GCM uncertainty was not assessed. However, Wilby et al. (2006) looked at changes in daily discharge for the Kennet (which flows into the Thames at Reading, UK) with three GCMs (using statistical downscaling) under two emissions scenarios (A2 and B2), and showed that the uncertainty due to choice of GCM is greater than that from emissions. The most thorough study so far, in terms of inclusion of the most sources of uncertainty, is probably that of Wilby and Harris (2006), who apply four GCMs, two emissions scenarios, two statistical downscaling techniques, two hydrological model structures and two sets of hydrological model parameters, in a study of the impact on low flows in the Thames. They conclude that the results are most sensitive to the choice of GCM and downscaling method, and less sensitive to the choice of hydrological model parameters or emissions scenario.

This study aims to provide a comparison of six sources of uncertainty, in terms of the impact of climate change on flood frequency. Section 2 outlines the basic methodology applied, including a brief description of the hydrological model, and presents the two catchments in England used for demonstration purposes. Section 3 describes the sources of uncertainty considered, and gives more detail on their precise application, whilst Section 4 presents results of the single-propagation of these sources through to the impact of climate change on flood frequency. It compares the size and position of the impact ranges for the different sources, and shows how these relate to natural variability. Multi-propagation is not attempted. Conclusions are given in Section 5. 


\section{Methodology}

\subsection{Hydrological model}

The main hydrological model used is the Probability Distributed Model (PDM; Moore 1985, 2007), which is a flexible, conceptual rainfall-runoff model, requiring inputs of catchment-average rainfall and potential evaporation. The model has been widely applied in Britain and forms part of the River Flow Forecasting System (Moore et al. 2005). The version used here has five catchment-specific parameters, four of which require calibration (the fifth is set using soils data). See Kay et al. (2007) for more detail on this version of the model, its parameters and calibration. A second hydrological model is used for the demonstration of impact uncertainty due to hydrological model structure. This is described in Section 3.4

\subsection{Catchments and baseline data}

The two example catchments, part of the UK National River Flow Archive (www.ceh.ac.uk/data/nrfa/), are catchment 40005 (the Beult at Stile Bridge, in South East England) and 74001 (the Duddon at Duddon Hall, in North West England). Their locations are shown on the map in Figure 1, and some details of the catchments are given in Table 1 . Both catchments are essentially rural, but are very different in terms of area, rainfall regime and topography. These factors, as well as location (amongst other things), mean a differing impact of climate change.

Hourly catchment average rainfall series are available for each catchment, for the period 1985-2001. These have been derived from data from all available daily raingauges within/near the catchments, with the sub-daily distribution determined by more limited amounts of quality-checked hourly rainfall data. Rainfall on days with no good quality hourly data is distributed using a set of catchment average profiles, derived using the average variability method (Pilgrim et al. 1969).

MORECS (Meteorological Office Rainfall and Evaporation Calculation System) monthly data (Thompson et al. 1982; Hough et al. 1996) are used to provide catchment potential evaporation (PE) inputs. These data are based on the Penman-Monteith equation for PE (Monteith, 1965) and are readily available as average values for 201 $40 \mathrm{~km} \times 40 \mathrm{~km}$ grid squares across Great Britain. The monthly PE for a catchment is produced by weighting the PE data for each MORECS grid square by the proportion of the catchment in that square, and then summing over the squares. The monthly values are then disaggregated equally down to the required input time-step of the model.

\subsection{Flood frequency derivation and application of climate change scenarios}

The generic method used here to estimate the impact of climate change on flood frequency is to drive the rainfall-runoff model with inputs (rainfall and PE data) representative of a) current and b) potential future climate conditions. Flood frequency curves are then derived from the resulting flow time-series, and the impact represented by the percentage change in flood magnitude (current to future) at several different return periods. Note that the flood magnitude with a return period of $\mathrm{T}$ years is that which might be expected to be equalled or exceeded, on average, once every $\mathrm{T}$ years, i.e. with an average interval of $\mathrm{T}$ years. 
In the derivation of flood frequency from time-series, peaks-over-threshold (POT) are preferred over annual maxima, as they make more use of the data (see Begueria (2005) for a discussion). Here, peaks are extracted at an average rate of three per year (i.e. the peak threshold is implicit), with standard independence criteria applied (Bayliss and Jones, 1992). The magnitudes of the POT are fitted using the generalised Pareto distribution with the peak arrival times assumed to correspond to a Poisson distribution (Naden, 1992), and fitting is carried out using the method of probability-weighted moments (Hosking and Wallis, 1987).

Here, the current climate conditions are generally those given by the (observed) baseline rainfall and PE data (for the 17-year period 1985-2001; Section 2.2), and the future climate conditions are given by adjusting the baseline data according to various climate change scenarios; often termed the 'delta change method'. That is, percentage changes in monthly mean rainfall (and PE) are derived from sets of current/future scenario data (e.g. from GCMs), and these are applied to the baseline data at each time-step (often daily or sub-daily). Variations on this simple application are clearly possible (Prudhomme et al. 2002), for example only enhancing the larger storms, but there is no right or wrong answer to how it should be done. Here then, the straightforward delta change method has been used in most cases, but some results using other downscaling methods are also presented, to demonstrate downscaling uncertainty (Section 3.3).

A major advantage of using the delta change method is the ease with which alternative emissions scenarios, or those based on alternative GCMs etc., can be applied. A disadvantage is that it is highly dependent on the variability and ordering of events within the (relatively short) baseline period, and does not allow for changes in variability (which can have a major impact, e.g. Arnell et al. 2003). This is explored in Section 3.6.

\subsection{Impact definition}

The impact of climate change on flood frequency is often defined by the percentage change in a flood peak of a given return period, but there is no single figure for the impact at any location as this is likely to differ by return period. The impact is also dependent on the future time-slice under consideration, as would be expected. However, not only is this dependence not necessarily linear, but the direction of change may not be consistent between time-slices: Under a given emissions scenario, downscaling method, hydrological model etc., some catchments can show an increase in flood frequency to the 2050s but a decrease by the 2080s, or vice-versa (Reynard et al. 2004). This is likely to be due to the balance between increased winter rainfall and decreased summer rainfall in the climate scenarios considered in that study, with higher temperatures and so increased evaporation, meaning higher soil moisture deficits which have to be refilled before flooding can occur. Only the change by the 2080s is considered here.

A further complication in the definition of the impact is the time-step or averaging period used to extract POT: the flood frequency curve based on hourly instantaneous flows will differ from that based on daily or monthly mean flows, and so too might the percentage changes in these curves from current to future time-slices. Here, the impact will be defined by the change in the flood magnitude at five return periods $(2,510,20$ 
and 50 years), where the flood frequency curve has been fitted to peaks extracted from hourly flow data.

Furthermore, it should be recognised that there is uncertainty in the fitting of a flood frequency curve to point data. This is particularly true where there are 'outlier' events in the point series, or where a catchment's flood events can be generated by very different processes (e.g. by seasonal variation in flood-production mechanisms) (Alila and Mtiraoui, 2002). It is for this reason that the flood frequency curve is not intended for extrapolation to significantly higher return periods, but simply to interpolate and smooth the point data. Note that the results presented here for the 50-year return period are a slight extrapolation, given the data availability for the example catchments, and should thus be treated with caution. Plotting the extracted peaks along with the fitted curve allows a visual assessment of the homogeneity of the points. A particular problem to bear in mind is the potential presence of 'outliers' in either the current or future flood peaks when RCM data are used directly, as their part-stochastic nature with initialcondition uncertainty (essentially a demonstration of natural variability) could generate extreme events which significantly affect the modelled change in flood frequency (Bell et al., 2007b). This problem would be solved by the availability of large RCM ensembles.

\section{Sources of uncertainty and their application}

\subsection{Future greenhouse gas emissions}

The UK Climate Impacts Programme (UKCIP) selected one emissions scenario from each of the four SRES storylines (IPCC, 2000), for their latest set of climate change scenarios for the UK (UKCIP02; Hulme et al. 2002). The selection was made so as to span the range of SRES scenarios well; for the A1 storyline, the highest emissions scenario was selected (A1F1). The emissions scenarios termed High, Medium-High, Medium-Low and Low in UKCIP02 correspond, respectively, to the SRES A1F1, A2, B2 and B1 emissions scenarios. However, the impact of these emissions on UK climate was only specifically modelled for a single scenario and time-slice, A2 (Medium-High) 2080s, using a three member ensemble of the Hadley Centre RCM HadRM3H; the impact under the other three emissions scenarios and additional time-slices was patternscaled from the A2 HadRM3H ensemble mean using the change in global temperature from the corresponding runs of the GCM, HadCM3.

The UKCIP02 scenarios are available as monthly (percentage or absolute) changes (from the 1961-1990 baseline) in various climate variables for a 50 x $50 \mathrm{~km}$ grid over the UK. Rainfall changes are directly available but the hydrological model also requires input time-series of potential evaporation (PE), which are not available directly from UKCIP02. The Penman-Monteith equation has thus been used to estimate changes in $\mathrm{PE}$ from those of other variables, for consistency with the baseline PE data (Section 2.2). This calculation involves temperature, wind speed, relative humidity and net surface long and short wave radiation, changes in which are available for the UKCIP02 scenarios. The simple delta change method (Section 2.3) is used here to apply the monthly percentage changes in rainfall and PE, derived from each UKCIP02 scenario, to the baseline hourly data for the catchments. 


\subsection{GCM structure}

There are a number of GCMs, developed and run in various countries across the globe, which generally project different climate changes when forced by a given emissions scenario. Not only do they have different climate sensitivities (the change in global mean temperature under a doubling of $\mathrm{CO}_{2}$ ) but they show different patterns of change in temperature and precipitation (see Figures 24-27 of Hulme et al. (2002) for patterns of change in winter and summer temperature and precipitation across the UK, under 9 different GCMs). Information on changes in various climate variables under future emissions scenarios, from a number of GCMs, was obtained from the IPCC data distribution centre (ipcc-ddc.cru.uea.ac.uk).

As for the UKCIP02 scenarios, PE is not a direct output of GCMs and so PenmanMonteith PE has been calculated from other variables for each of the GCMs. Note though that not all of the GCMs provide directly usable variables for the calculation of PE, so substitutes sometimes have to be used. Even when the required variables are available, the calculated monthly PE may not seem realistic, possibly due to errors resulting from the coarse resolution of the GCMs.

The 5 GCMs represented here are HadCM3 (UK), CSIRO-Mk2 (Australia), CGCM2 (Canada), ECHAM4 (Germany) and CCSR (Japan). These are a subset of the models that contributed to the IPCC Third Assessment Report (IPCC 2001); the others each lack some of the data required for this application. The delta change method is used to apply monthly percentage changes in rainfall and PE, derived from each of the GCMs, to the baseline hourly data for the catchments.

GCMs also use parameterisations, to deal with processes that occur on scales smaller than the grid resolution of the GCM (e.g. schemes to estimate the amount of cloud). Some of these schemes are well-constrained (by observations) but others are less well understood, hence the uncertainty due to parameterisation could be important (Murphy et al. 2004). However, GCM parameterisation uncertainty is not covered here due to a lack of readily-available data from a perturbed physics ensemble at the time of writing. Work in this area is evolving quickly though, as demonstrated by New et al. (2007) who use initial results from the climateprediction.net experiment (Stainforth et al. 2005) to demonstrate that the uncertainty from this perturbed-physics ensemble dominates that from hydrological model uncertainty, when modelling changes in the median flow of the Thames at Teddington.

\subsection{Downscaling from GCMs}

The coarse spatial resolution of GCMs, and the greater uncertainty in their outputs at fine temporal resolution, means that they are generally not appropriate for finer scale impacts modelling, like flooding. However, the more recent use of RCMs, nested within GCMs, provides dynamic downscaling to a more appropriate temporal and spatial scale (especially for precipitation; Durman et al. 2001). Previously the delta change method was often used as an alternative to direct use of GCM data (e.g. Prudhomme et al. 2002), and this is applied here alongside other downscaling methods, including direct use of RCM data, to demonstrate downscaling uncertainty. 


\subsubsection{Delta change versus direct use of RCM data}

The first comparison is between three variations on the delta change method and the direct use of data from an RCM. Two of the delta change methods use different data sources to determine the monthly percentage changes that are applied; the first uses GCM (HadCM3) data while the second uses RCM (UKCIP02/HadRM3H) data (that is, including dynamic downscaling from the GCM scale down to the RCM scale). These changes are applied to the baseline hourly rainfall and PE through the simple application of the monthly percentage changes to each hour of baseline data according to month. However, this does not allow for more complex changes in rainfall distributions. Reynard et al. (2004) developed an extended version of the delta change method which aims to match changes in daily rainfall intensity as well as percentage changes in monthly means (PE is still adjusted simply through changes in monthly means), and it is this which is applied in the third version of the delta change method, again using changes derived from UKCIP02 RCM data.

Briefly, this extended delta change method uses an indicator of the change in frequency of the 20-year return period rainfall for each of the four seasons, and the precise way in which the monthly percentage changes in rainfall are applied depends upon the value of the corresponding seasonal indicator. For instance, an increase in mean rainfall for a certain month is applied differently if there is also a significant increase in the 20-year return period rainfall (when larger storms will be further enhanced) than if there is a significant decrease (when larger storms will be reduced).

The final of the four methods uses data directly from an RCM (25km HadRM3H) to derive rainfall and PE inputs for the hydrological model (Kay et al. 2006a, b). This $\mathrm{RCM}$ is the same as that used for UKCIP02, but with a further improvement in the temporal and spatial resolution (hourly precipitation data on a $\sim 25 \mathrm{~km}$ grid). The RCM was driven by the same boundary conditions used for the first member of the UKCIP02 SRES A2 ensemble i.e, providing a rerun of this experiment at double the horizontal resolution. Application here involves the direct use of RCM data for both current (19611990) and future (2071-2100) time-slices, looking at changes between flood frequency results for the two periods, as bias in the RCM data means that it is generally not appropriate to consider changes from an observed flood frequency curve.

Other studies have attempted to correct for bias in RCM data by deriving linear error factors from a comparison of current time-slice data with observed data (on annual, seasonal or monthly bases), and then applying these factors to the future time-slice data before feeding it into an impact model (e.g. Graham et al. 2007a, Thodsen 2007, Fowler and Kilsby 2007). However, care must be taken when applying such methods, especially for flood frequency estimation: Leander and Buishand (2007) show that simple linear bias-correction can lead to worse reproduction of flood frequency than no correction at all, and instead apply a nonlinear power transformation. Graham et al. (2007b) caution that '...one must keep in mind the alterations made to the RCM results, and the important assumption that RCM model biases in the future are systematically the same as in the control climate. The more scaling applied, the further away one gets from "direct" use of the RCM'. No scaling has been applied to the $25 \mathrm{~km}$ resolution RCM data used here. 


\subsubsection{RCM structure}

The second comparison is between different dynamic downscaling models, here represented by different RCMs nested in the same GCM. These have been obtained from the PRUDENCE project (Christensen et al. 2007), which produced high-resolution climate change scenarios for Europe (for 2071-2100) based on the use of different RCMs nested within the Hadley Centre GCM HadAM3H (essentially reruns of UKCIP02 experiments with different RCMs). Eight RCMs are used, from the Hadley Centre (UK), DMI (Denmark), ETH (Switzerland), GKSS (Germany), MPI (Germany), SMHI (Sweden), UCM (Spain) and KNMI (Netherlands). Jacob et al. (2007) and Deque et al. (2007) provide brief descriptions of each of these RCMs, with further references from which more detail can be obtained, along with descriptions of model performance for present-day climate and of uncertainties in model projections for temperature and precipitation. Included in this comparison is the more recent Hadley Centre RCM, HadRM3P driven by (the high resolution version of) HadAM3P (Jones et al. 2008) in a series of experiments parallel to those run for UKCIP02. HadAM3P is an updated version of HadAM3H providing some improvements in its global climatology but with similar global and European responses to climate change.

For each of these RCMs, changes in mean monthly rainfall can be calculated directly from the monthly time-series of rainfall available for the control and scenario timeslices (2080s, A2 emissions). However, the calculation of changes in mean monthly PE involves an initial calculation of monthly time-series of PE from monthly time-series of other meteorological variables, using the Penman-Monteith formulation as for UKCIP02 data. Here, the variables used were generally those of temperature, wind speed, dew point temperature and net surface long and short wave radiation, but for some RCMs specific humidity had to be used as dew point temperature was not available. The simple delta change method has been used to apply the changes calculated from each RCM to baseline catchment rainfall and PE.

Note that one PRUDENCE RCM (that of ICTP (Italy)) could not be used due to lack of available wind speed data, meaning that Penman-Monteith PE could not be calculated. Alternative, simpler, formulations of PE could have been applied (Oudin et al. 2005), in particular one of a number involving just temperature in the calculation. However, in a climate change context, the inclusion of other meteorological variables could be critical in terms of changes in them affecting the changes in PE. Arnell (1999) investigated the sensitivity of modelled changes in runoff, under a given climate change scenario, to PE formulation, on a European scale. He compared two PE formulae, Penman-Monteith and Priestly-Taylor (the latter of which does not specifically include humidity and wind speed), and found that, whilst giving similar estimates of current runoff, they predicted differing changes in runoff, even of different directions in a number of regions (including South-East England).

For the set of RCMs used here, for catchment 40005 in south-east England, changes in PE calculated using the purely temperature-based formula suggested by Oudin et al. (2005) range between $+13 \%$ (spring) and $+26 \%$ (winter) whereas those calculated using the Penman-Monteith formulation range between $-16 \%$ (autumn) and $+75 \%$ (summer). Thus the range of changes is greatly increased when other variables are included, and the seasonal distribution of changes also differs. This could certainly affect water 
resource impacts, but could also affect flood impacts through changes in the balance of rainfall and PE. An initial investigation using these two PE formulations suggests that the effect here is relatively small compared to other sources of uncertainty (not shown), but it is still important to use a consistent formulation for calculating PE throughout the analysis. The calculation of PE from climate model data, and the resulting hydrological uncertainty, will be explored further in future work. One potential problem could be the reliability of the additional variables required for the calculation Penman-Monteith PE, when these are derived from RCMs. This is discussed by Ekstrom et al. (2007) with regard to data from HadRM $3 \mathrm{H}$.

\subsection{Hydrological model structure}

There are numerous hydrological models, each of which could be used to assess the effect of climate change on flows. No model is perfect in its representation of reality, and the choice of model must be based on, for instance, study aims, performance under current conditions for required catchments/areas, data requirements etc.

Here, results from the 5-parameter PDM (Section 2.1) will be compared to those from a second model, the Grid-to-Grid (G2G; Bell et al. 2007a). The latter is a grid-based runoff and routing model, requiring gridded inputs of rainfall and potential evaporation, which has been formulated on a $1 \mathrm{~km}$ grid over the UK. Its parameters are determined through the use of spatial data sets alongside either catchment or area-wide calibration; area-wide calibrations have been used here.

Both the PDM and the G2G models have been driven directly with data from an hourly RCM (25km HadRM3H) for current (1961-1990) and future (2071-2100, A2 emissions scenario) time-slices. Information on the finer-scale spatial variation of rainfall (via the use of standard annual average rainfall data available on a $1 \times 1 \mathrm{~km}$ grid over Britain) is used to convert data from the $25 \mathrm{~km} \mathrm{RCM}$ grid to the catchment average rainfall required by the PDM (see Kay et al. 2006b), or to the $1 \mathrm{~km}$ gridded rainfall required by the G2G (see Bell et al. 2007a). Any difference in the results from the two models is therefore predominantly due to inherent differences in model structure, rather than input data.

\subsection{Hydrological model parameters}

Hydrological model parameters that require calibration will be uncertain. A different method of fitting, or using a different measure of fit (objective function), will likely result in different calibrated parameter values. This is particularly true where there is any sort of interdependence between parameters. In addition, data quality, for both input data (rainfall and potential evaporation) and calibration data (observed flows), could affect calibration performance, as could data quantity. There must be a sufficient length of data, covering a range of flow regimes, for calibration to be effective. The calibrated parameters will thus differ to some extent according to the data used for calibration. Uncertainty in the observed flow data used for calibration, especially for high flows, should also be borne in mind.

There are various ways in which calibration uncertainty can be systematically investigated. The method applied here, for the PDM, is a variation on the statistical technique of jack-knifing (Shao and Tu, 1995). This involves the generation of a 
number of different calibrated parameter sets, each based on slightly different data: one whole year of observed flow data is ignored (set as missing) in the generation of each set (all input data are retained, to preserve the year-to-year water balance). Thus $\mathrm{N}$ years of data leads to $\mathrm{N}+1$ calibrated parameter sets (one based on the use of all the data). Each set has then been used with the baseline input data and with inputs adjusted by the delta change method (UKCIP02 Medium-High 2080s), and the change in flood frequency calculated for each pair at specific return periods. Error bars are then constructed by estimating the variance $\left(\sigma^{2}\right)$ from the values of the jack-knifed flood frequency changes at each return period. The 95\% error bars can then be plotted as $\mu \pm 2 \sigma$, where $\mu$ is the mean of the jack-knifed values. However, jack-knife theory requires that the variance be calculated slightly differently to a usual sample (with a multiplier of $(\mathrm{N}-1) / \mathrm{N}$ rather than $1 / \mathrm{N})$, and this inflates the size of the error bars.

\subsection{Internal variability of the climate system}

All of the above sources of uncertainty derive from our representation of the physical systems we are modelling and the drivers of change in those systems. In each case, the uncertainty is assessed by either applying different representations of current and future climates to a hydrological model or one representation of current and future climate to different hydrological models. Due to the natural internal variability of the climate system, the representation used of each of these climates will not be unique. Using an alternative, equally valid, representation would produce different results. It is thus important that we explore the range of different hydrological responses that could be obtained by our finite sampling of the internal variability of the climate system. This will allow us to assess the importance of the other sources of uncertainty.

Two ways of assessing the effect of climate variability are explored. The first uses the model-based approach of running an ensemble of climate model integrations started from different initial conditions, with the climate model and its forcings identical in each integration. Each then evolves a different but plausible evolution of the climate and sampling from these allows us to explore the implication of internal variability. For UKCIP02 this was done using a three-member ensemble (see Figures 24-27 of Hulme et al. 2002). To demonstrate this source of uncertainty here, data were obtained from the EU-funded PRUDENCE project (Christensen et al. 2007; http://prudence.dmi.dk), for the Hadley Centre RCM HadRM3P nested in a three-member initial condition ensemble of the Hadley Centre GCM HadAM3P (2080s, A2 emissions scenario). The delta change method is used to apply monthly percentage changes in rainfall and PE, derived from each RCM experiment in the ensemble, to the baseline hourly data for the catchments. It should be noted however that such model-based climate variability ensembles only give a modelled estimate of natural variability, which may or may not be representative of real variability (Section 7.7 of Hulme et al. 2002) and which will differ between GCMs (e.g. Ruosteenoja et al. 2003). Also, it is too computationally expensive to run very large GCM/RCM ensembles.

One alternative for assessing the effect of climate variability is to use some form of stochastic rainfall model to generate a large number of rainfall time-series for the current or future climates (e.g. Cameron 2006, Kilsby et al. 2007). However, such use would require significant checking of the performance of the rainfall model under current conditions, in terms of its ability to simulate extremes at the required time-step 
as well as its replication of the seasonal cycle etc. In addition, there is usually an assumption that features of the model fitted to the current climate are still valid under a future climate.

Here then, the second way used to explore the effect of climate variability is to apply a simple and pragmatic resampling of rainfall series to produce a large number of new rainfall series. Monthly resampling involves the formation of new time-series through the random selection of rainfall, month by month, from the original series. For example, from a baseline series for January 1985 - December 2000, a new series would be created by first selecting a January from any of those in the baseline period, to represent January 1985, then selecting any February to represent February 1985, and so on until a series of the same length as the original is created. The months are selected with replacement, so that the rainfall for the same month could be repeated in any one of the resampled series, and some months may not be used at all. Resampling by month or season (3month blocks) limits the effect of time-correlation (autocorrelation) in rainfall series (e.g. dependence of daily rainfall on the rainfall of preceding days). It does not allow for variation in the short term extremes (e.g. hourly/daily maxima etc.), but does allow variation in longer term accumulations by, for instance, meaning that a wet winter can potentially follow a wet autumn (which may not have occurred within the original time series). Thus, in addition to creating plausible new rainfall series, this approach addresses an issue with the delta change method of downscaling, which is highly dependent on the variability and ordering of events within the (relatively short) baseline period. In particular, the sequencing of wet and dry seasons and years could have a significant effect on the flood frequency, so the resampling method essentially demonstrates the potential effect of natural variability in antecedent conditions. The method will not, however, address the issue of the effects of multi-decadal variability (Kendon et al. 2008), which the initial condition climate model ensemble approach can address. Resampling thus represents a simple proxy for the effect of medium-frequency natural variability.

Resampling under the current climate allows the representativeness of the original baseline series to be assessed, by comparison against the median and bounds from the set of resampled series. Resampling under the current and future climates allows the potential range due to natural variability under the current climate to be compared to the range of changes that might be expected under climate change. Accordingly, a set of 100 resampled rainfall series have been produced for both the current and future climate, with resampling in 3-month blocks, and the model run with each new rainfall series. The future climate uses the simple delta change method to adjust the baseline rainfall and $\mathrm{PE}$ according to the UKCIP02 medium-high emissions scenario for the 2080s. The median flood frequency curve and its upper and lower $90 \%$ bounds have then been calculated for each return period.

\section{Results}

\subsection{Natural variability}

Assessing the importance of natural variability from resampling observed rainfall indicates it has a significant influence for both catchments, but larger (in terms of percentage changes) for catchment 40005, particularly at higher return periods (Table 2). The smaller effect for catchment 74001 could be due to the fact that the resampling 
method only allows variation in longer term extremes (seasonal rainfall amounts), as the longer memory of larger, flatter catchments, like 40005, means that variation in longer term extremes has a greater effect on them than on more responsive (small, steep) catchments, like 74001. In contrast, variation of shorter term extremes would have a greater effect on more responsive catchments.

However, the effect of climate change could be much more significant, in comparison with natural variability, for catchment 74001 as its 'Future' bounds are consistently shifted upwards compared to the 'Current' bounds, whereas the 'Future' bounds for catchment 40005 are almost completely contained within its 'Current' bounds (Figure 2). This is consistent with the results of Hulme et al. (1999), who looked at the potential changes in mean annual runoff over Europe by the 2050s and compared these to natural variability. They found a band across central Europe, including southern Britain, where the effect of climate change was less than that of natural variability, whereas regions further north or south had a more pronounced climate change effect compared to natural variability. For both of our catchments though, natural variability could be a significant factor in the future experience of flooding, with or without climate change (the potential, versus the actual). Hulme et al. (1999) point out that, for some sectors, adapting management systems to withstand natural climate variability may be sufficient to also withstand climate change, in some regions and for the medium-term, but this may not be sufficient in other regions or for the longer term.

The results for current and future natural variability using resampling are used in Figure 3 (plotted as median with $90 \%$ error bars) to provide context for the ranges of impacts of climate change on flood frequency from the other sources of uncertainty described in Section 3. The ranges are shown at five different return periods $(2,5,10$, 20 and 50 years) and are calculated as the percentage change from the simulated baseline flood frequency (i.e. that simulated using the observed input data) except for the downscaling example using RCM data directly, where the change is calculated from the RCM-simulated baseline (see Section 3.3.1). The figure distinguishes between sets (different sources of uncertainty) but does not indicate which member of each set is which (although this is discussed to some extent below). However, the results demonstrating uncertainty from hydrological model parameters are shown by the mean and the $95 \%$ error bars, rather than as a separate point for each jack-knifed parameter set (see Section 3.5).

Included in Figure 3 are the results for future natural variability using the GCM/RCM initial condition ensemble (see Section 3.6). These are consistent with those for future natural variability using resampling, and are generally well-contained within the ranges of the latter. The exception is lower return periods ( $<10$ years) for catchment 40005 , where the impact from one ensemble member is slightly above the upper $90 \%$ bound from the resampling. The ordering of the effect of the three ensemble members differs between the two catchments, although the third ensemble member generally has the greatest effect on both (at least at lower return periods).

\subsection{Uncertainty ranges from physical system models and their drivers}

The other results presented in Figure 3 indicate that: 
- Uncertainty due to emissions is very low for catchment 40005 , but more important for catchment 74001.

- GCM uncertainty could be quite important for both catchments, although the GCM resulting in the highest increase in flood frequency for both catchments, CCSR, is quite extreme (compared to the other GCMs used) in terms of the increase in winter rainfall that it predicts for the UK (see Figures 26 and 28 of Hulme et al. 2002). The ordering of the effect of other GCMs differs between the two catchments.

- The three delta change downscaling methods show very similar results, with a decrease in flood frequency at higher return periods for catchment 40005 , and an increase in flood frequency at all return periods for catchment 74001. Results from the direct use of RCM data show similar decreases for 40005 and increases for 74001 .

- The results for the eight RCMs show a similar pattern of change, with an increase at lower return periods and a decrease (or lower percentage increase) at higher return periods for catchment 40005, whilst for catchment 74001 there is an increase at most return periods which is larger for higher return periods. However, the ordering of the impacts from the eight RCMs is different for each catchment, with the UCM RCM generally showing the greatest effect for catchment 40005 (although the GKSS and MPI RCMs take over at higher return periods) but the MPI RCM showing the greatest effect (at all return periods) for catchment 74001. Similarly, the DMI RCM generally shows the least effect for catchment 40005 while the Hadley RCM shows the least effect (at all return periods) for catchment 74001.

- The two hydrological models compare very well, and show the same pattern of a decrease in flood frequency change with return period for catchment 40005 , and a more uniform flood frequency change with return period for catchment 74001.

- There is a very small effect from hydrological model parameter uncertainty.

\subsection{Comparison of uncertainty ranges}

The ranges of impact shown in Figure 3 overlap to a good extent, but their sizes vary quite considerably. This is illustrated in Figure 4, which shows bar charts of the impact range sizes based on the data points plotted in Figure 3.

When all of the data points are included, GCM structure is the dominant source of uncertainty (Figure 4a). However, when the data points for the most extreme GCM (CCSR) are excluded, the uncertainty due to GCM structure becomes more similar in size to that from other sources of uncertainty (Figure 4b). Although the uncertainty from hydrological model parameters is seemingly the smallest in most cases, the ordering of the other sources varies by return period and between the two catchments.

Table 3 shows the ordering of the sources when the size of the impact range is averaged over the five illustrated return periods (when the CCSR GCM is excluded, Figure 4b; 'GCM structure' is promoted to number 1 in the list if this GCM is included). The more dominant sources of uncertainty in this case appear to be those related to the GCM or to the method of downscaling from the GCM (including RCM structure). The uncertainty from hydrological model structure is higher for catchment 40005 than for catchment 74001 probably because of the G2G's lower performance for catchments with a less topographically-driven flow regime (Bell et al. 2007a), particularly with the area-wide parameters used here (rather than catchment-calibrated parameters). 
The impact ranges from GCMs and RCMs with different structure were derived using data from single integrations of each climate model. As demonstrated with the application of data from the GCM initial condition ensemble, the range of GCM structure uncertainty could be narrowed or widened by accounting for natural variability. This will also be the case with the range of RCM structure uncertainty, at least in terms of the influence of fine-scale climate variability. (In general the RCMs add fine-scale information to a large-scale solution mainly determined by the driving GCM thus it will be this aspect of climate variability that is relevant to the RCM structure uncertainty range.) Thus when comparing the GCM and RCM-derived ranges the estimates of uncertainty due to natural variability provide an estimate of the uncertainty in the magnitude of each range.

The application of a simple resampling technique showed that natural variability in antecedent conditions or seasonal climate could be important for both of the example catchments and potentially even more important than climate change (at least up to the 2080s) for catchment 40005. The size of the potential range of natural variability under current conditions is actually comparable with the larger ranges from the various sources of climate change uncertainty: natural variability would appear at the top of the list in Table 3 for catchment 40005 (second for catchment 74001), although the full range of uncertainty from GCM structure exceeds that of natural variability for both catchments. The positioning of the future changes relative to the bounds from current natural variability is the crucial factor though, as is illustrated in Figure 2 and Figure 3.

\section{Conclusions}

This paper has discussed sources of uncertainty in climate change impact studies, with particular reference to the impact of climate change on flood frequency in Britain. Examples were given of the single-propagation of the sources of uncertainty through to their range of impacts on flood frequency, for two catchments in England. Multipropagation (that is, propagation of more than one source of uncertainty at once) was not attempted, although this is what would be required for a full risk-based approach to decision-making, along with estimated likelihoods of the different combinations.

The results from single-propagation of each of the sources of uncertainty suggested that uncertainty from GCM structure was the dominant source of uncertainty. However, this is due to the extremely large increases in winter rainfall predicted by one of the 5 GCMs used (CCSR). Omitting the results for this GCM led to other sources of uncertainty becoming more significant, although uncertainty from sources relating to modelling of the future climate was generally still larger than that relating to emissions or hydrological modelling. Natural variability could also play a significant role, and the uncertainty due to natural variability should be considered when comparing the uncertainties from the physical system models and their drivers.

The results presented here are not conclusive as some of the sources of uncertainty are not fully represented. For example

- The range of emissions scenarios used (from UKCIP02) is not the full IPCC range (and the A1F1, B1 and B2 scenarios are scaled from the ensemble results for the A2 scenario). 
- Only five GCMs are used to represent GCM structure uncertainty. More exist, but there are difficulties either in terms of data availability or in the calculation of PE for these. Also, ideally, the same RCM would be nested within each different GCM, to give the added-value of finer temporal and spatial resolution whilst demonstrating GCM structure uncertainty in a consistent way.

- The use of simple delta change downscaling (Section 2.3) is likely to mean an underestimation of the range of uncertainties simulated from RCM structure, for example, as this method of downscaling concentrates on changes in mean climate and does allow for more complex changes in variability, which may differ between RCMs.

- Only two models are used to represent hydrological model structure uncertainty, one of which (the G2G) has particular difficulty in flatter regions (Bell et al. 2007a). Ongoing development of the $\mathrm{G} 2 \mathrm{G}$ should improve its performance in such regions.

- The uncertainty from hydrological model parameters is being represented through the use of jack-knifed calibrated parameter sets, which cover the uncertainty from data but not from equifinality (Beven 2001) etc. Thus the full effect of parameter uncertainty on flood frequency estimation is probably larger than that represented here.

- Resampling in 3-month blocks is used as a simple proxy for natural variability, mainly by allowing for natural variability in antecedent conditions. Use of methods which allow for variation in shorter term extremes would likely result in wider ranges of natural variability, particularly for more responsive catchments (like 74001).

Additions would not necessarily increase the range of uncertainty from any source, but would lead to more robust conclusions.

More catchments need to be studied to determine anything conclusive about the importance of different sources of uncertainty; whether overall or for different types of catchment or different locations. However, the ordering here is consistent with the conclusions of studies of uncertainty in the impact of climate change on water resources (Arnell 1999, Prudhomme and Davies 2008, Wilby et al. 2006, Wilby and Harris 2006, Graham et al. 2007a, b) which each concluded that uncertainty from global climate modelling is generally larger than that from other sources. Ideally though, ensemble experiments using each GCM / RCM would be used to generate impact ensembles. Taking the mean impact for each would thus limit the 'noise' from natural variability that is present within these uncertainty ranges. The use of an RCM perturbed-physics ensemble is to be explored in the next phase of this research, as is the use of transient RCM runs (covering the period 1950 to 2100). The latter will enable a study of the detectability of climate change impacts on river flows against the background of (modelled) natural variability. The detectability of climate change impacts amongst natural variability is a problem which needs to be tackled so that policy-makers can be more readily convinced of the need to factor climate change into their long-term plans at an early stage (Wilby 2006).

\section{Acknowledgements}

Data from a number of GCMs were obtained from the IPCC data distribution centre (http://ipcc-ddc.cru.uea.ac.uk). Data from a number of RCMs were obtained through the PRUDENCE data archive (http://prudence.dmi.dk), funded by the EU through contract 
EVK2-CT2001-00132. This work was funded via the UK Met Office Hadley Centre by the Joint Defra and MoD Programme; (Defra) GA01101 (MoD) CBC/2B/0417_Annex C5.

\section{References}

Alila, Y., and Mtiraoui, A. (2002). Implications of heterogeneous flood-frequency distributions on traditional stream-discharge prediction techniques. Hydrological Processes, 16, 1065-1084.

Arnell, N.W. (1999). The effect of climate change on hydrological regimes in Europe: a continental perspective. Global Environmental Change, 9, 5-23.

Arnell, N.W. (2003). Relative effects of multi-decadal climatic variability and changes in the mean and variability of climate due to global warming: future streamflows in Britain. Journal of Hydrology, 270, 195-213.

Arnell, N.W., D. Hudson, and R.G. Jones (2003): Climate change scenarios from a regional climate model: Estimating change in runoff in southern Africa. J. Geophys. Res., 108(D16), 4519, doi:10.1029/2002JD002782.

Bayliss, A. and Jones, R. (1992). The peaks-over-threshold database at the Institute of Hydrology. Report to UK Ministry of Agriculture, Fisheries and Food, Institute of Hydrology, Wallingford, 74pp.

Begueria, S. (2005). Uncertainties in partial duration series modelling of extremes related to the choice of the threshold value. Journal of Hydrology, 303, 215-230.

Bell, V.A., Kay, A.L., Jones, R.G. and Moore, R.J. (2007a). Development of a high resolution grid-based river flow model for use with regional climate model output. Hydrology and Earth System Sciences, 11, 532-549.

Bell, V.A., Kay, A.L., Jones, R.G. and Moore, R.J. (2007b). Use of a grid-based hydrological model and regional climate model outputs to assess changing flood risk. International Journal of Climatology, 27(12), 1657-1671.

Beven, K.J. (2001). Calibration, validation and equifinality in hydrological modelling. In: Anderson, M.G. and Bates, P.D. (eds) Model validation: Perspectives in Hydrological Science, 43-44, Wiley, Chichester.

Booij, M.J. (2005). Impact of climate change on flooding assessed with different spatial model resolutions. Journal of Hydrology, 303, 176-198.

Cameron, D. (2006). An application of the UKCIP02 climate change scenarios to flood estimation by continuous simulation for a gauged catchment in the northeast of Scotland, UK (with uncertainty). Journal of Hydrology, 328, 212-226. 
Cameron, D., Beven, K. and Naden, P. (2000). Flood frequency estimation by continuous simulation under climate change (with uncertainty). Hydrology and Earth System Sciences, 4, 393-405.

Christensen, J.H., Carter, T.R., Rummukainen, M. and Amanatidis, G. (2007) Evaluating the performance and utility of regional climate models: the PRUDENCE project. Climatic Change, 81, 1-6.

Déqué, M., Rowell, D. P., Lüthi, D., Giorgi, F., Christensen, J. H., Rockel, B., Jacob, D., Kjellström, E., de Castro, M. and van den Hurk, B. (2007) An intercomparison of regional climate simulations for Europe: assessing uncertainties in model projections. Climatic Change, 81, 53-70.

Durman, C.F., Gregory, J.M., Hassell, D.C., Jones, R.G., Murphy, J.M., 2001. A comparison of extreme European daily precipitation simulated by a global and a regional climate model for present and future climates. Quarterly Journal of the Royal Meteorological Society, 127 (573), 1005-1015.

Ekstrom, M., Jones, P.D., Fowler, H.J., Lenderink, G., Buishand, T.A. and Conway, D. (2007). Regional climate model data used within the SWURVE project 1: projected changes in seasonal patterns and estimation of PET. Hydrology and Earth System Sciences, 11(3), 1069-1083.

Fowler, H.J. and Kilsby, C.G. (2007). Using regional climate model data to simulate historical and future river flows in northwest England. Climatic Change, 80, 337-367.

Graham, L.P., Andreasson, J. and Carlsson, B. (2007a). Assessing climate change impacts on hydrology from an ensemble of regional climate models, model scales and linking methods - a case study on the Lule River basin. Climatic Change, 81, 293-307.

Graham, L.P., Hageman, S., Jaun, S. and Beniston, M. (2007b). On interpreting hydrological change from regional climate models. Climatic Change, 81, 97-122.

Hosking, J.R.M. and Wallis, J.R. (1987). Parameter and quantile estimation for the Generalised pareto distribution. Technometrics, 29, 339-349.

Hough, M., Palmer, S., Weir, A., Lee, M., and Barrie, I. (1996). The Meteorological Office Rainfall and Evaporation Calculation System: MORECS version 2.0 (1995). An update to Hydrological Memorandum 45, The Met. Office, Bracknell.

Hulme, M., Barrow, E.M., Arnell, N.W., Harrison, P.A., Johns, T.C. and Downing, T.E. (1999). Relative impacts of human-induced climate change and natural climate variability. Nature, 397, 688-691.

Hulme, M., Jenkins, G.J., Lu, X.,Turnpenny, J.R., Mitchell, T.D., Jones, R.G., Lowe, J., Murphy, J.M., Hassell, D., Boorman, P., McDonald, R. and Hill, S. (2002). Climate Change Scenarios for the United Kingdom: The UKCIP02 Scientific Report. Tyndall 
Centre for Climate Change Research, School of Environmental Sciences, University of East Anglia, Norwich, UK.

IPCC (2000). Special report on emissions scenarios (SRES): A special report of Working Group III of the Intergovernmental Panel on Climate Change. Cambridge University Press, Cambridge.

IPCC (2001). Climate Change 2001: The Scientific Basis. Cambridge University Press, Cambridge.

Jacob, D., Barring, L., Christensen, O. B., Christensen, J. H., de Castro, M., Deque, M., Giorgi, F., Hagemann, S., Hirschi, M., Jones, R., Kjellström, E., Lenderink, G., Rockel, B., Sanchez, E., Schär, C., Seneviratne, S. I., Somot, S., van Ulden, A., and van den Hurk, B. (2007), An inter-comparison of regional climate models for Europe: Design of the experiments and model performance, Climatic Change, 2007, 81, 31-52.

Jenkins, G. and Lowe, J. (2003). Handling uncertainties in the UKCIP02 scenarios of climate change. Hadley Centre technical note 44.

Jones R.G., Murphy J.M., Hassell D.C., Woodage M.J. (2008) A high resolution atmospheric GCM for the generation of regional climate scenarios. Submitted to Climate Dynamics.

Kay, A.L., Jones, D.A., Crooks, S.M., Kjeldsen, T.R. and Fung, C.F. (2007). An investigation of site-similarity approaches to generalisation of a rainfall-runoff model. Hydrology and Earth System Sciences, 11, 500-515.

Kay, A.L., Jones, R.G. and Reynard, N.S. (2006a). RCM rainfall for UK flood frequency estimation. II. Climate change results. Journal of Hydrology, 318, 163-172.

Kay, A.L., Reynard, N.S. and Jones, R.G. (2006b). RCM rainfall for UK flood frequency estimation. I. Method and validation. Journal of Hydrology, 318, 151-162.

Kendon, E.J., Rowell, D.P., Jones, R.G. and Buonomo, E. (2008). Robustness of future changes in local precipitation extremes. J. Climate, 21(17), 4280-4297 doi: 10.1175/2008JCLI2082.1.

Kilsby, C.G., Jones, P.D., Burton, A., Ford, A.C., Fowler, H.J., Harpham, C., James, P., Smith, A. and Wilby, R.L. (2007). A daily weather generator for use in climate change studies. Environmental Modelling and Software, 22(12), 1705-1719.

Leander, R. and Buishand, T.A. (2007). Resampling of regional climate model output for the simulation of extreme river flows. Journal of Hydrology, 332, 487-496.

Monteith, J.L., (1965). Evaporation and environment. Symposia of the Society for Experimental Biology, 19, 205-234. 
Moore, R.J. (1985). The probability-distributed principle and runoff production at point and basin scales. Hydrolog. Sci. J., 30, 273-297.

Moore, R.J. (2007). The PDM rainfall-runoff model. Hydrology and Earth System Sciences, 11, 483-499.

Moore, R.J., Bell, V.A. and Jones, D.A. (2005). Forecasting for flood warning. C.R. Geoscience, 337, 203-217.

Murphy, J.M., Sexton, D.M.H., Barnett, D.N., Jones, G.S., Webb, M.J., Collins, M. and Stainforth, D.A. (2004). Quantification of modelling uncertainties in a large ensemble of climate change simulations. Nature, 430, 768-772.

Naden, P.S. (1992). Analysis and use of peaks-over-threshold data in flood estimation. In: A.J. Saul (Ed.) Floods and Flood Management, Kluwer Academic, Dordrecht, 131143.

New, M., Lopez, A., Dessai, S. and Wilby, R. (2007). Challenges in using probabilistic climate change information for impact assessments: an example from the water sector. Philosophical Transactions of the Royal Society A, 365, 2117-2131.

Oudin, L., Hervieu, F., Michel, C., Perrin, C., Andreassian, V., Anctil, F. and Loumagne, C. (2005). Which potential evapotranspiration input for a lumped rainfallrunoff model? Part 2 - Towards a simple and efficient potential evapotranspiration model for rainfall-runoff modelling. Journal of Hydrology, 303, 290-306.

Pilgrim, D.H., Cordery, I. and French, R. (1969). Temporal patterns of design rainfall for Sydney. Civil Engineering Transactions, Institution of Engineers, Australia, CE11, 9-14.

Prudhomme, C. and Davies, H.N. (2008) Assessing uncertainties in climate change impact analyses on river flow regimes in the UK. Part 2: future climate. Clim Change, doi: 10.1007/s10584-008-9461-6.

Prudhomme, C., Reynard, N. and Crooks, S. (2002). Downscaling from global climate models for flood frequency analysis: where are we now? Hydrological Processes, 16, $1137-1150$.

Reynard, N.S., Crooks, S.M. and Kay, A.L. (2004). Impact of climate change on flood flows in river catchments. Report to the UK Department for Environment, Food and Rural Affairs and the Environment Agency, Project SC010011 technical report, CEH Wallingford, March 2004, 97pp.

Rowell, D.P. (2006). A demonstration of the uncertainty in projections of UK climate change resulting from regional model formulation. Climatic Change, 79, 243-257.

Ruosteenoja, K., Carter, T.R., Jylhä, K. and Tuomenvirta, H. (2003). Future climate in world regions: an intercomparison of model-based projections for the new IPCC 
emissions scenarios. The Finnish Environment 644, Finnish Environment Institute, Helsinki.

Shao, J. and Tu, D. (1995). The Jackknife and Bootstrap. Springer, New York.

Stainforth, D. A., Aina, T., Christensen, C., Collins, M., Faull, N., Frame, D.J., Kettleborough, J.A., Knight, S., Murphy, J.M., Piani, C., Sexton, D., Smith, L.A., Spicer, R.A., Thorpe, A.J. and Allen, M.R. (2005). Uncertainty in predictions of the climate response to rising levels of greenhouse gases. Nature, 433, 403-406.

Thodsen, H. (2007). The influence of climate change on stream flow in Danish rivers. Journal of Hydrology, 333, 226-238.

Thompson, N., Barrie, I.A. and Ayles, M. (1982). The Meteorological Office Rainfall and Evaporation Calculation System: MORECS (July 1981). Hydrological Memorandum No. 45, Met Office, Bracknell.

Wilby, R.L. (2005). Uncertainty in water resource model parameters used for climate change impact assessment. Hydrol. Processes, 19(16), 3201-3219.

Wilby, R.L. (2006). When and where might climate change be detectable in UK river flows? Geophysical Research Letters, 33, L19407, doi:10.1029/2006GL027552.

Wilby R.L. and Harris, I. (2006). A framework for assessing uncertainties in climate change impacts: Low-flow scenarios for the River Thames, UK. Water Resources Research, 42, W02419, doi:10.1029/2005WR004065.

Wilby, R.L., Whitehead, P.G., Wade, A.J., Butterfield, D., Davis, R.J. and Watts, G. (2006). Integrated modelling of climate change impacts on water resources and quality in a lowland catchment: River Kennet, UK. Journal of Hydrology, 330, 204-220. 


\section{List of Figures}

Figure 1 Map showing the locations of the two example catchments.

Figure 2 Examples of flood frequency uncertainty from natural variability, showing results from resampled baseline (solid) and future (dotted) rainfall. The median flood frequency curve and the upper and lower 90\% bounds are shown for each return period, from 100 resampled series (3-month blocks). The simple delta change method is applied for the future scenario (2080s, UKCIP02 medium-high emissions). The flood frequency simulated with observed rainfall (squares/dashed line) is also shown.

Figure 3 Graphs of the variation in the impact of climate change on flood frequency, from various sources, for five return periods $(2,5,10,20$ and 50 years). The impact is shown as the percentage change in flood frequency from the current period to the 2080s. The potential ranges of current and future natural variability from resampling are also shown for comparison (from Table 2), by bars at the median and at the $90 \%$ upper and lower bounds.

Figure 4 Bar charts showing the relative size of the impact range from the various scenarios and methods, a) for all the possibilities presented previously, and b) after excluding the results for the most extreme GCM (CCSR). The results are shown for 5 return periods $(2,5,10,20$ and 50 years) and as means over those return periods. 


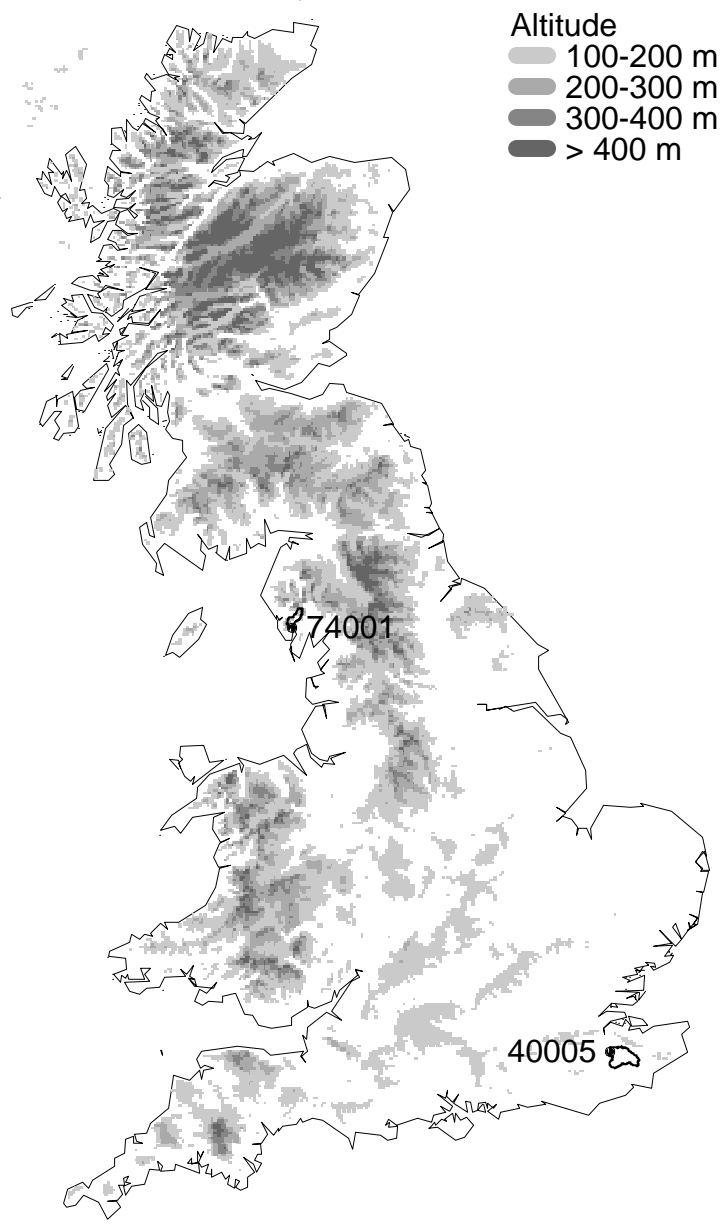

Figure 1 Map showing the locations of the two example catchments. 


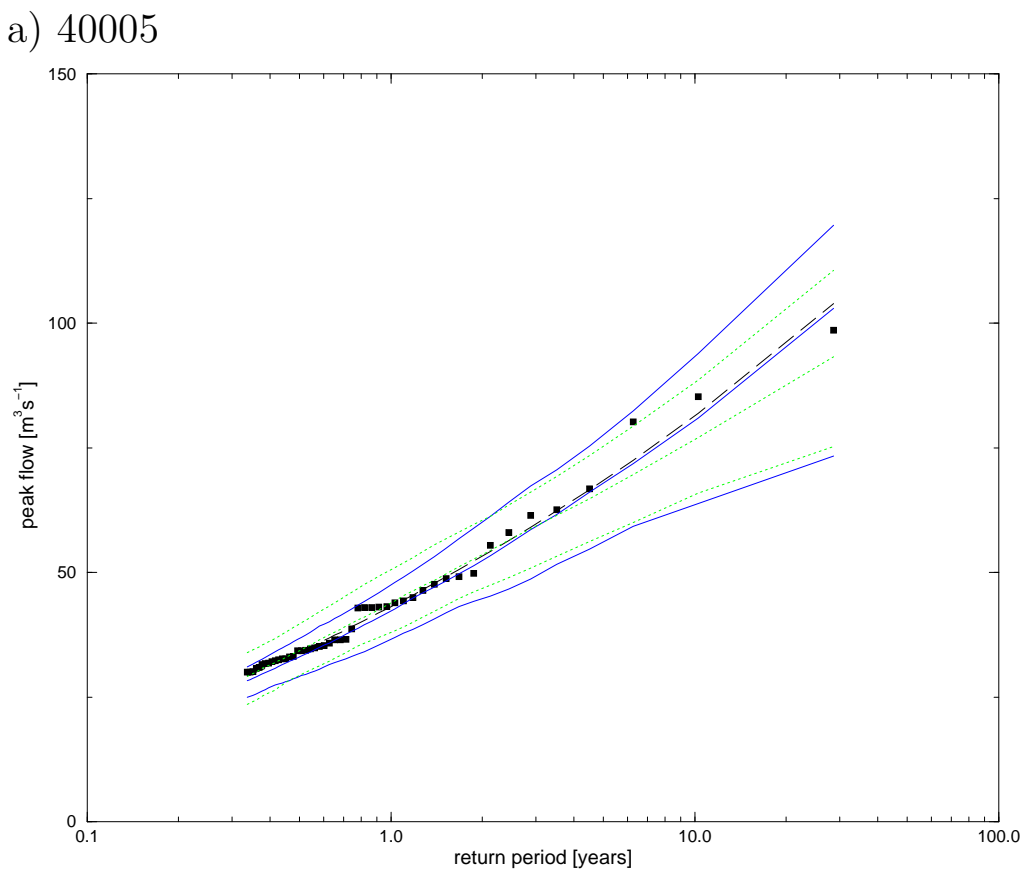

b) 74001

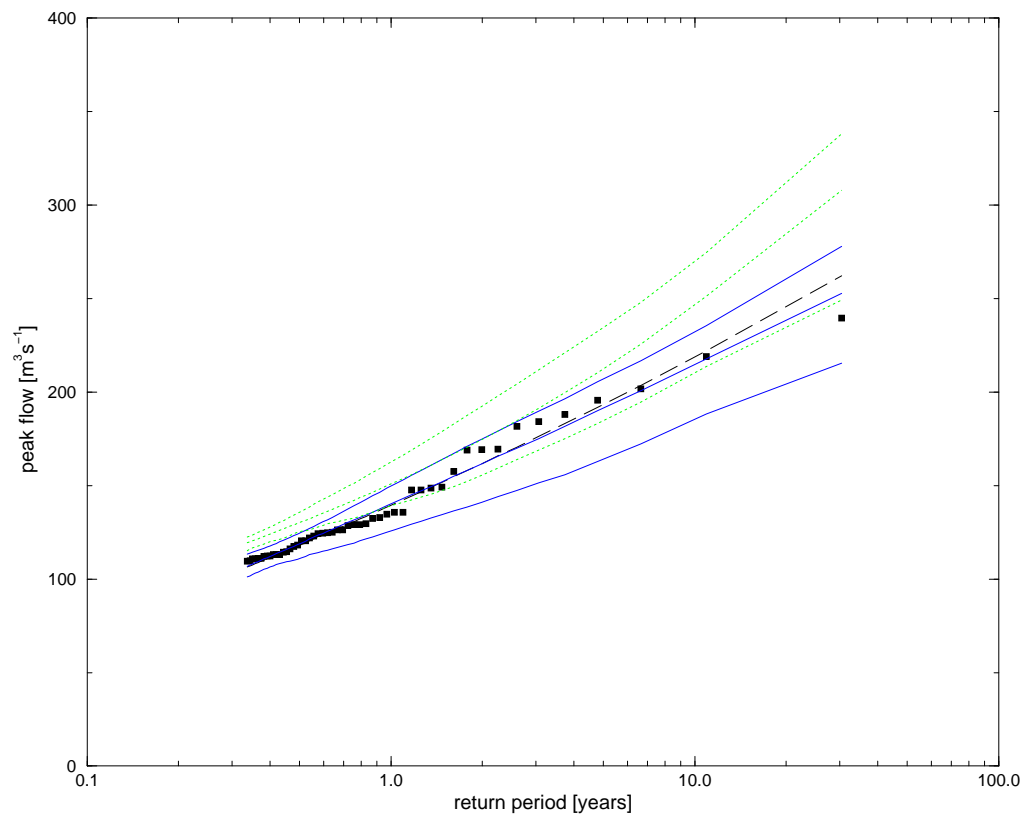

Figure 2 Examples of flood frequency uncertainty from natural variability, showing results from resampled baseline (solid blue) and future (dotted green) rainfall. The median flood frequency curve and the upper and lower $90 \%$ bounds are shown for each return period, from 100 resampled series (3-month blocks). The simple delta change method is applied for the future scenario $(2080 \mathrm{~s}$, UKCIP02 medium-high emissions). The flood frequency simulated with observed rainfall (squares/dashed line) is also shown. 
a) 40005

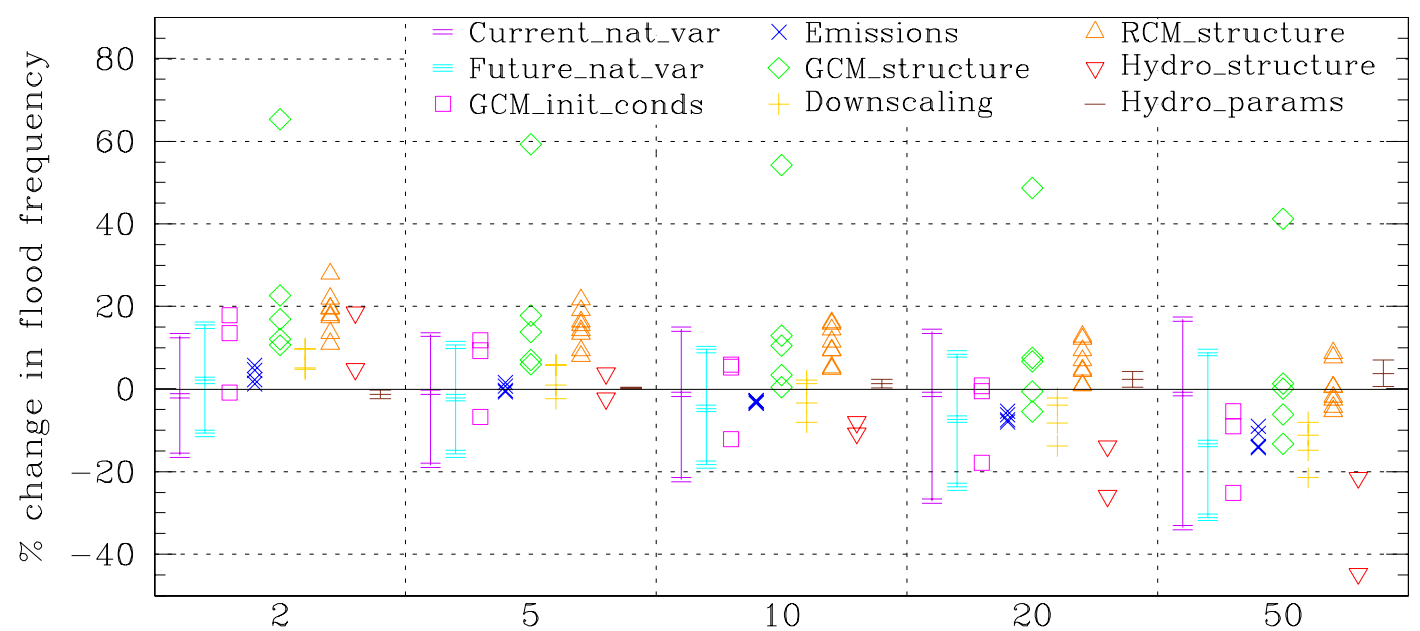

b) 74001

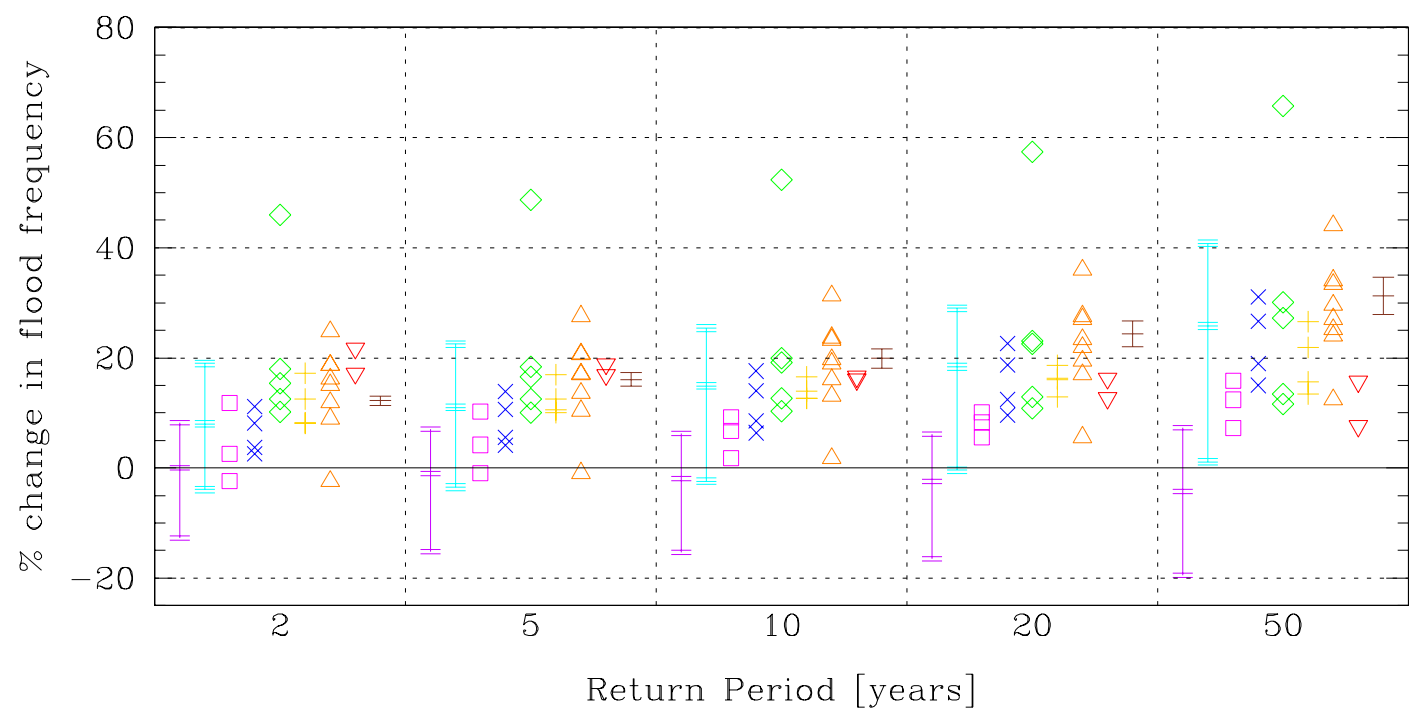

Figure 3 Graphs of the variation in the impact of climate change on flood frequency, from various sources, for five return periods $(2,5,10,20$ and 50 years). The impact is shown as the percentage change in flood frequency from the current period to the $2080 \mathrm{~s}$. The potential ranges of current and future natural variability from resampling are also shown for comparison (from Table 2), by bars at the median and at the $90 \%$ upper and lower bounds. 
a)
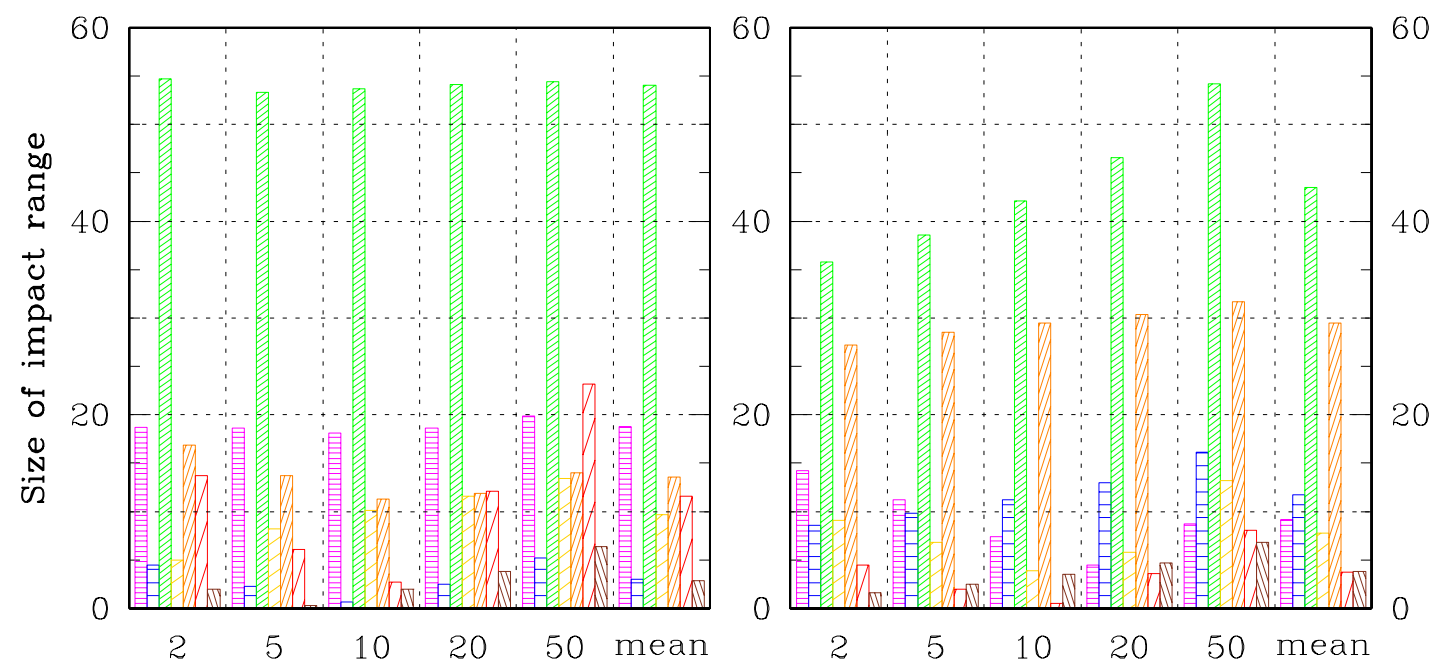

b)

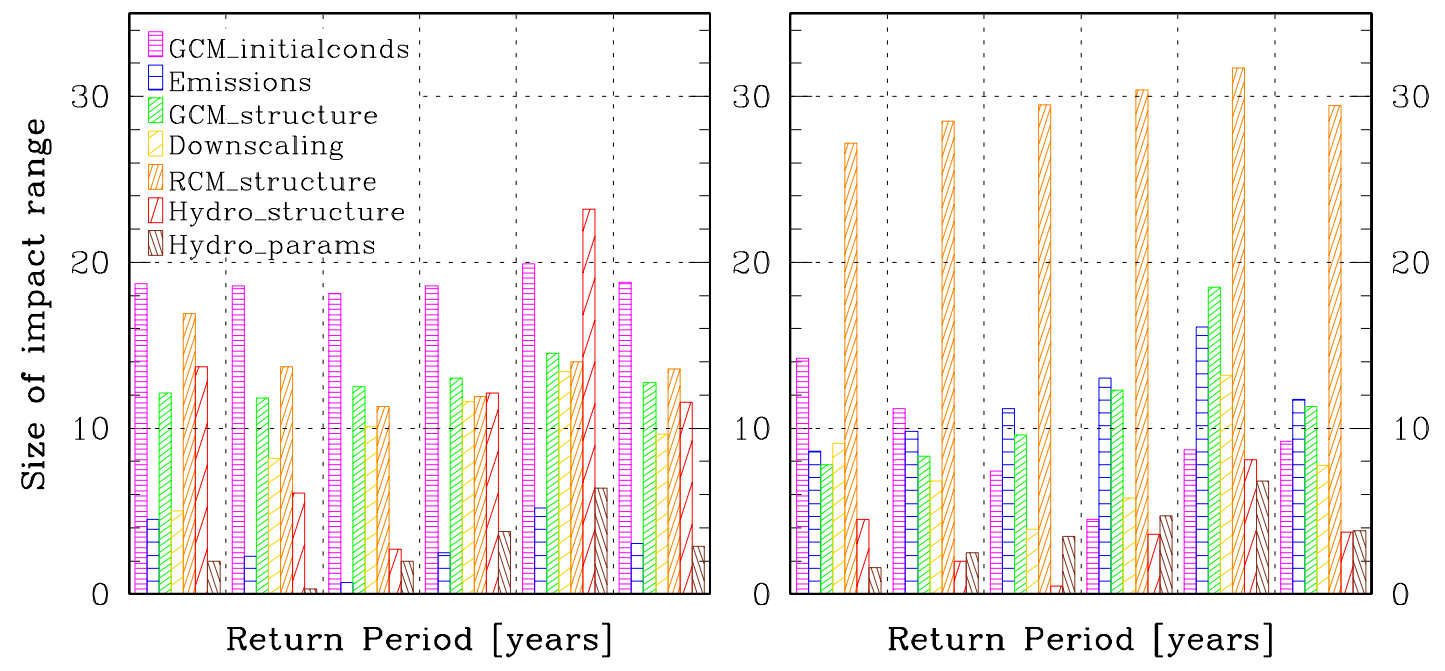

Figure 4 Bar charts showing the relative size of the impact range from the various scenarios and methods, a) for all the possibilities presented previously, and b) after excluding the results for the most extreme GCM (CCSR). The results are shown for 5 return periods $(2,5,10,20$ and 50 years $)$ and as means over those return periods. 


\section{List of Tables}

Table 1 Details of the two example catchments.

Table 2 Description of the resampling results for the current and future periods, for the median flood frequency curve and the upper and lower $90 \%$ bounds.

Table 3 The ordering of the sources of uncertainty for the example catchments, based on the size of their impact ranges averaged over the five return periods (excluding the CCSR GCM).

Table 1 Details of the two example catchments.

\begin{tabular}{crlrrrrr}
\hline $\begin{array}{c}\text { Catchment } \\
\text { number }\end{array}$ & $\begin{array}{c}\text { Catchment } \\
\text { area }\left(\mathrm{km}^{2}\right)\end{array}$ & $\begin{array}{l}\text { Altitude } \\
\text { range }(\mathrm{m})\end{array}$ & $\begin{array}{c}\text { Mean } \\
\text { altitude } \\
(\mathrm{m})\end{array}$ & $\begin{array}{c}\text { Baseflow } \\
\text { index }\end{array}$ & $\begin{array}{c}\text { Mean } \\
\text { flow } \\
\left(\mathrm{m}^{3} \mathrm{~s}^{-1}\right)\end{array}$ & $\begin{array}{c}\mathrm{SAAR}_{61-90} \\
(\mathrm{~mm})\end{array}$ & $\mathrm{R}$ \\
\hline 40005 & 277 & $13-161$ & 45 & 0.24 & 2.1 & 690 & 0.34 \\
74001 & 86 & $17-799$ & 315 & 0.28 & 4.8 & 2265 & 0.81 \\
\hline
\end{tabular}

SAAR $_{61-90}=$ standard annual average rainfall for $1961-1990, \mathrm{R}=$ mean annual runoff $/$ mean annual rainfall. 
Table 2 Description of the resampling results for the current and future periods, for the median flood frequency curve and the upper and lower $90 \%$ bounds.

\begin{tabular}{|c|c|c|c|c|c|c|c|}
\hline \multirow{2}{*}{ Catchment } & \multirow{2}{*}{$\begin{array}{l}\text { Period } \\
\text { (change } \\
\text { from...) }\end{array}$} & \multirow{2}{*}{$\begin{array}{l}\% \text { change } \\
\text { to... }\end{array}$} & \multicolumn{5}{|c|}{ Return Period } \\
\hline & & & 2 & 5 & 10 & 20 & 50 \\
\hline \multirow[t]{8}{*}{40005} & \multirow{4}{*}{$\begin{array}{l}\text { Current } \\
\text { (from } \\
\text { simulated } \\
\text { baseline) }\end{array}$} & \multirow{4}{*}{$\begin{array}{l}90 \% \text { lower } \\
\text { median } \\
90 \% \text { upper } \\
\text { size of range } \\
\text { (upper-lower) }\end{array}$} & -16.0 & -18.4 & -21.9 & -27.1 & -33.5 \\
\hline & & & -1.7 & -0.8 & -1.3 & -1.3 & -1.2 \\
\hline & & & 12.9 & 13.2 & 14.5 & 14.0 & 16.9 \\
\hline & & & 28.9 & 31.6 & 36.4 & 41.1 & 50.4 \\
\hline & \multirow{4}{*}{$\begin{array}{l}\text { Future } \\
\text { (from } \\
\text { median } \\
\text { Current) }\end{array}$} & \multirow{4}{*}{$\begin{array}{l}90 \% \text { lower } \\
\text { median } \\
90 \% \text { upper } \\
\text { size of range } \\
\text { (upper-lower) }\end{array}$} & -10.7 & -15.7 & -18.3 & -23.7 & -31.1 \\
\hline & & & 2.1 & -2.1 & -4.7 & -7.3 & -13.2 \\
\hline & & & 15.5 & 10.7 & 9.6 & 8.5 & 8.8 \\
\hline & & & 26.1 & 26.4 & 27.9 & 32.2 & 39.9 \\
\hline \multirow[t]{8}{*}{74001} & \multirow{4}{*}{$\begin{array}{l}\text { Current } \\
\text { (from } \\
\text { simulated } \\
\text { baseline) }\end{array}$} & $90 \%$ lower & -12.7 & -15.2 & -15.3 & -16.5 & -19.5 \\
\hline & & median & 0.0 & -1.0 & -1.9 & -2.4 & -4.3 \\
\hline & & $90 \%$ upper & 8.2 & 7.1 & 6.3 & 6.1 & 7.3 \\
\hline & & $\begin{array}{l}\text { size of range } \\
\text { (upper-lower) }\end{array}$ & 20.9 & 22.3 & 21.5 & 22.6 & 26.7 \\
\hline & \multirow{4}{*}{$\begin{array}{l}\text { Future } \\
\text { (from } \\
\text { median } \\
\text { Current) }\end{array}$} & \multirow{4}{*}{$\begin{array}{l}90 \% \text { lower } \\
\text { median } \\
90 \% \text { upper } \\
\text { size of range } \\
\text { (upper-lower) }\end{array}$} & -3.9 & -3.5 & -2.4 & -0.4 & 1.1 \\
\hline & & & 8.0 & 11.0 & 14.9 & 18.4 & 25.8 \\
\hline & & & 19.0 & 22.5 & 25.4 & 29.0 & 40.8 \\
\hline & & & 22.9 & 25.9 & 27.8 & 29.4 & 39.7 \\
\hline
\end{tabular}

Note: For the 'Current' period the percentage differences are shown from the flood frequency simulated with the original baseline rainfall. For the 'Future' period the percentage differences are shown from the median current flood frequency.

Table 3 The ordering of the sources of uncertainty for the example catchments, based on the size of their impact ranges averaged over the five return periods (excluding the CCSR GCM).

\begin{tabular}{lll}
\hline & 40005 & 74001 \\
\hline 1 & GCM initial conditions & RCM structure \\
2 & RCM structure & Emissions \\
3 & GCM structure & GCM structure \\
4 & Hydro' structure & GCM initial conditions \\
5 & Downscaling & Downscaling \\
6 & Emissions & Hydro' parameters \\
7 & Hydro' parameters & Hydro' structure \\
\hline
\end{tabular}

